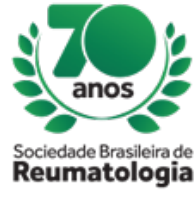

\title{
CLINICAL AND EPIDEMIOLOGICAL PROFILE OF PATIENTS WITH OSTEOPOROSIS WHO ARE IN USE OF TERIPARATIDE AT A RHEUMATOLOGY SERVICE OF A UNIVERSITY HOSPITAL
}

Sergio Henrique Oliveira dos Santos (Hospital Universitário Getúlio Vargas, Manaus, AM, Brasil), Juliana Bühring (Hospital Universitário Getúlio Vargas, Manaus, AM, Brasil), Guilherme Andrade Bubol (Hospital Universitário Getúlio Vargas, Manaus, AM, Brasil), Fernanda Maria da Silva Bezerra (Hospital Universitário Getúlio Vargas, Manaus, AM, Brasil), Rosana Barros de Souza (Hospital Universitário Getúlio Vargas, Manaus, AM, Brasil), Sandra Lúcia Euzébio Ribeiro (Hospital Universitário Getúlio Vargas, Manaus, AM, Brasil)

\section{BACKGROUND}

Osteoporosis (OP) is characterized by low bone mineral density (BMD), skeletal fragility and changes in bone microarchitecture raising the incidence of osteoporotic fractures, which in turn, represents an important health problem, since they increase the risk for some kinds of fractures such as hip and vertebral ones that are associated with increased morbidity and mortality. Teriparatide $20 \mathrm{mcg}$ once daily for 18 to 24 months has showed reduction of vertebral and non-vertebral fractures, as well as increase of the BMD T-score. Our objectives were to describe the epidemiological and clinical profile of the patients at Rheumatology Service from a University Hospital with diagnosis of osteoporosis in use of teriparatide and to check the BMD L1-L4 and femoral neck (FN) T-score variation (DT-score) after teriparatide treatment has instituted.

\section{MATERIALS AND METHODS}

Follow-up protocols of patients with diagnosis of osteoporosis were reviewed, and the inclusion criteria were the usage of teriparatide and the presence of pre and post-treatment BMD test. Correlation between DT-score and teriparatide usage time was checked through the Pearson correlation coefficient (r).

\section{RESULTS}

Three hundred and twenty one patients with osteoporosis are followed up, 23 (7.16\%) started teriparatide, only 12 (3.74\%) women in menopause have met the inclusion criteria, 11 were excluded because incomplete data. Of these, four (33.3\%) have used teriparatide for more than 18 months. Mean age at diagnosis was 63.8 years, standard deviation (SD) 10.5 years, $6(50 \%)$ had previous history of lowimpact fracture, 7 (58\%) osteoarthritis and $6(50 \%)$ diagnosis of essential hypertension (EH). In pretreatment BMD when considering L1-L4 segment, mean density was $0.656 \mathrm{~g} / \mathrm{cm} 2, \mathrm{SD} 0.116 \mathrm{~g} / \mathrm{cm} 2$, T-score -4 , SD 0.923; the FN density was $0.588 \mathrm{~g} / \mathrm{cm} 2$, SD $0.134 \mathrm{~g} / \mathrm{cm} 2$, T-score -3.1, SD 1.27 . In post-treatment BMD L1-L4 segment mean density was $0.740 \mathrm{~g} / \mathrm{cm} 2$, SD $0.119 \mathrm{~g} / \mathrm{cm} 2$, T-score -3.5 , SD 1; in FN density was $0.643 \mathrm{~g} / \mathrm{cm} 2, \mathrm{SD} 0.095 \mathrm{~g} / \mathrm{cm} 2$ and T-score -2.6 , SD 0.77 . Mean time usage was approximately 15.4 months SD of 4.2 months. There was a positive correlation between DT-score in L1-L4 with $r=0.603(p=0.038)$, but not in DT-score of FN with $r=0.241(p=0.451)$.

\section{CONCLUSION}

All patients who started in teriparatide usage were women in menopause; the majority had osteoarthritis, half, essential hypertension and previous history of low-impact fracture. There was a strong positive correlation only between DT-score in L1-L4 as a function of treatment time, but we found no correlation between DT-score in FN as a function of treatment time. 